ATMOSPHERIC CHEMISTRY

\title{
Just add water dimers
}

\section{Fast reaction with water dimers may limit the impact of the simplest Criegee intermediate on atmospheric chemistry}

\section{By Mitchio Okumura}

A

lkenes constitute a large fraction of the natural and human-made volatile organic compounds (VOCs) that are emitted into the troposphere. Their oxidation products degrade air quality and contribute to climate warming. Alkene oxidation is thought to involve Criegee intermediates (CIs), highly reactive molecules that form when ozone reacts with alkenes. However, the impact of CIs may be limited if they react rapidly with water. Modelers have found it difficult to quantify the effect of CIs on atmospheric composition, because laboratory data on CI reactions with water have been contradictory. On page 751 of this issue, Chao et al. (1) show that the simplest CI, formaldehyde oxide $\left(\mathrm{CH}_{2} \mathrm{OO}\right)$, reacts rapidly with the water dimer, $\left(\mathrm{H}_{2} \mathrm{O}\right)_{2}$. Similar results are reported by Lewis et al. (2).

Alkene ozonolysis is a common reaction in organic chemistry (see the figure) (3). Alkenes colliding with ozone initially form a primary ozonide, which rapidly dissociates to produce a stable ketone or aldehyde and a carbonyl oxide: the Criegee intermediate. This highly unusual molecule is both very reactive and highly polar. During ozonolysis, some CIs are formed hot and decay or react immediately, but a sizable fraction becomes stabilized. Scientists have long sought to determine the yields of stabilized CIs as well as their reactivity $(4,5)$, but the measurements were hampered by an inability to directly detect the elusive intermediate in the gas phase.

In 2008, Taatjes et al. reported the successful detection of a CI-formaldehyde oxide, $\mathrm{CH}_{2} \mathrm{OO}-$ in the gas phase with photoionization spectroscopy using vacuum ultraviolet synchrotron radiation (6). In 2012, they showed that relatively high concentrations of CIs could be generated in a short pulse by photolyzing diiodomethane, $\mathrm{CH}_{2} \mathrm{I}_{2}$, to form $\mathrm{CH}_{2} \mathrm{I}$, which reacts with $\mathrm{O}_{2}$ to form $\mathrm{CH}_{2} \mathrm{OO}$ in a gentle, nearly thermoneutral reaction (7). These two discoveries led to a rapidly growing literature that includes detection of larger CIs, discovery of a strong ultraviolet absorption band, and determination

Arthur Amos Noyes Laboratory of Chemical Physics, California Institute of Technology, Pasadena, CA 91125, USA. E-mail: mo@caltech.edu

of a number of reaction rate coefficients $(8,9)$.

Experiments that directly observed CIs found far higher reaction rates with atmospheric species such as $\mathrm{NO}_{2}, \mathrm{SO}_{2}$, and organic acids than did earlier studies. The fast reaction with $\mathrm{SO}_{2}$, which oxidizes very slowly in the atmosphere, led modelers to propose that CIs contribute substantially to sulfate aerosol formation in the troposphere (10).

The evidence for the reaction of CIs with water has been contradictory. End-product studies done over a decade ago concluded that CIs are lost with high yields at high relative humidities in environmental chambers. These experiments led atmospheric chemists to assume that CIs are rapidly lost at moderate to high relative humidities. However, more recent laboratory experiments found that CIs react slowly, if at all, with water molecules $(7,11,12)$.

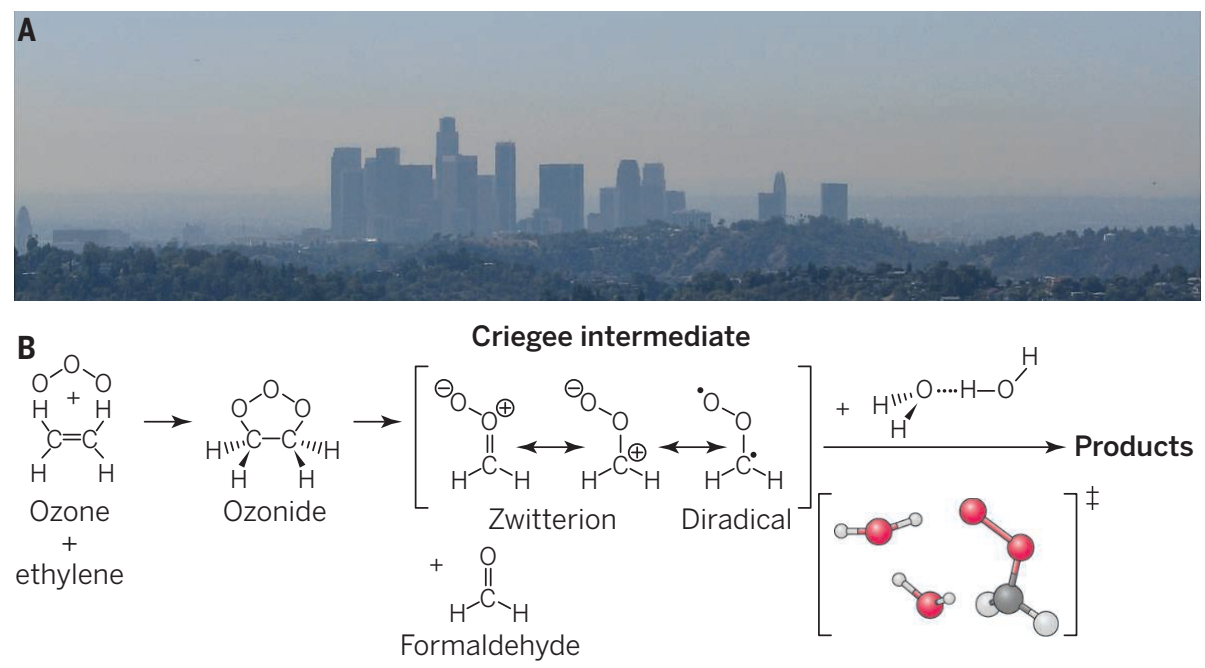

Alkene oxidation. (A) Alkenes such as ethylene, emitted by both plants and human sources, are oxidized in the atmosphere through reaction with ozone, degrading air quality. (B) During ethylene ozonolysis, a cyclic primary ozonide decomposes to form the simplest Criegee intermediate (formaldehyde oxide, $\mathrm{CH}_{2} \mathrm{OO}$ ) and formaldehyde. Two studies (1, 2) show that $\mathrm{CH}_{2} \mathrm{OO}$ reacts rapidly with water dimers. The molecular model shows the predicted transition state (13).

In 2004, Ryzhkov and Ariya proposed that CIs react not with isolated water molecules but rather with water dimers (13). On the basis of quantum chemistry and statistical rate calculations, they predicted that the water dimer would react $3.5 \times 10^{5}$ times as quickly as the monomer. The enhancement stems from the cooperativity of cyclic hydrogen-bonded structures, which preferentially stabilize po- lar transition states (see the figure), an effect well known from water cluster studies.

Experimental support for enhanced reactivity of the water dimer with CIs comes from experiments reported in 2014. Berndt et al. used a relative rate approach to show that $\mathrm{CH}_{2} \mathrm{OO}$ reacts with water dimers at a fast rate that depends quadratically on relative humidity (14). However, their rates exceeded upper bounds set in two experiments that used $\mathrm{CH}_{2} \mathrm{I}_{2}$ chemistry $(11,12)$.

Chao et al. and Lewis et al. have now succeeded in measuring the reaction rate of the simplest $\mathrm{CI}, \mathrm{CH}_{2} \mathrm{OO}$, with water vapor over a wide range of relative humidities. Both groups use the $\mathrm{CH}_{2} \mathrm{I}_{2}$ chemistry and take advantage of the strong UV absorption spectrum discovered by Beames et al. (9) to follow the decay of $\mathrm{CH}_{2} \mathrm{OO}$ in real time at much higher pressures and larger relative humidities than is possible with photoionization detection. Both groups find that the decay rate depends quadratically on the water vapor concentration-evidence that the CIs are reacting with the water dimer. By using the known equilibrium constant for the water dimer, they arrive at similar rate coefficients for the bimolecular reaction of $\mathrm{CH}_{2} \mathrm{OO}$ with $\left(\mathrm{H}_{2} \mathrm{O}\right)_{2}$. Chao et al. recorded more precise data over a wider range of relative humidity,

systematic errors may arise from estimated water concentration or missing secondary reactions.

The new studies $(1,2)$ validate the observation of rapid water dimer reaction by Berndt et al. (14), although the new rate constants are smaller by a factor of 1.5 to 2 . The results are consistent with the upper bound found by photoionization detection 
(7) at very low water concentrations, but lie well above the upper bounds set by the two other experiments that used $\mathrm{CH}_{2} \mathrm{I}_{2}$ chemistry $(10,11)$. The latter measurements inferred CI concentrations by monitoring secondary products as proxies, which might lead to erroneous results if other reactions form the same products.

The observed rates are so fast that, in the troposphere, reaction with the water dimer will be the largest sink for $\mathrm{CH}_{2} \mathrm{OO}$ and will limit its steady-state concentration. Of course, even small concentrations could conceivably still oxidize very stable species such as $\mathrm{SO}_{2}$. Previous simulations, such as those by Vereecken et al. (15), have assumed that water reactions are important but have been hampered by the large uncertainties in the rate coefficients. The atmospheric impact of this simplest of CIs can now be quantitatively addressed in model calculations.

Perhaps the most critical remaining issue concerns the reaction rates of larger CIs with water dimers. Quantum chemistry calculations predict that larger CIs react more slowly than $\mathrm{CH}_{2} \mathrm{OO}$, at least with one water molecule. Vereecken et al., among others, have predicted that the larger CIs could influence atmospheric composition, for example, by causing a substantial increase in sulfate aerosol loading $(8,10,15)$. However, the estimated rates could be off by orders of magnitude. Fortunately, the same approach used in the current papers can be applied to the larger CIs; indeed, Chao et al. present preliminary data that clearly reveal at least one conformer of acetaldehyde oxide, $\mathrm{CH}_{3} \mathrm{CHOO}$, reacting quickly with water dimers (1). The impact of the full range of CIs on atmospheric composition remains an open question, but one that experiments can now address.

\section{REFERENCES}

1. W. Chao, J-T. Hsieh, C.-H. Chang, J.J.-M. Lin Science 347 , 751(2015)

2. T. R. Lewis, M. A. Blitz, D. E. Heard, P.W. Seakins, Phys Chem. Chem Phys. 10.1039/C4CP04750H (2015).

3. R. Criegee, Angew. Chem. Int. Ed. Engl. 14,745 (1975).

4. D. Johnson, G. Marston, Chem. Soc. Rev. 37,699 (2008).

5. N. M. Donahue, G. T. Drozd, S. A. Epstein, A. A. Presto, J. H. Kroll, Phys. Chem. Chem. Phys. 13,10848(2011).

6. C. A. Taatjes et al., J. Am. Chem. Soc. 130,11883 (2008).

7. O.Welzetal., Science 335,204 (2012).

8. C. A. Taatjes, D. E. Shallcross, C. J. Percival, Phys. Chem. Chem. Phys. 16,1704 (2014)

9. J. M. Beames, F. Liu, L. Lu, M. I. Lester, J. Am. Chem. Soc. 134,20045 (2012).

10. C. J. Percival et al., Faraday Dis. 165,45 (2013).

11. B. Ouyang, M. W. McLeod, R. L. Jones, W. J. Bloss, Phys Chem. Chem. Phys. 15, 17070 (2013)

12. D. Stone, M. Blitz, L. Daubney, N. U. M. Howes, P. Seakins, Phys. Chem Chem Phys 16,1139(2014).

13. A. B. Ryzhkov, P. A. Ariya, Phys. Chem. Chem. Phys. 6,5042 (2004).

14. T. Berndtetal., Phys. Chem. Chem. Phys. 16, 19130 (2014).

15. L. Vereecken, H. Harder, A. Novelli, Phys. Chem. Chem. Phys. 16, 4039 (2014).

ACKNOWLEDGMENTS

Supported by NSF grant CHE-1413712.

10.1126/science.aaa5506

\section{Harnessing weak interactions for enantioselective catalysis}

\section{The traditional tools of physical organic chemistry benefit from modern data analysis techniques}

\section{$B y$ Tongxiang Lu and Steven E. Wheeler}

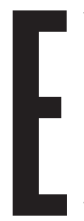

lucidating catalytic reaction mechanisms is often a challenge, and these difficulties are compounded in the case of enantioselective catalysts. The ability of a catalyst to preferentially form one enantiomer over the other often hinges on the balance of many attractive and repulsive nonbonded interactions that occur in competing transition states. On page 737 of this issue, Milo et al. (1) combine physical organic and computational quantum chemistry with modern data analysis techniques to identify these interactions. Their predictive mathematical models elucidate the underlying reac-

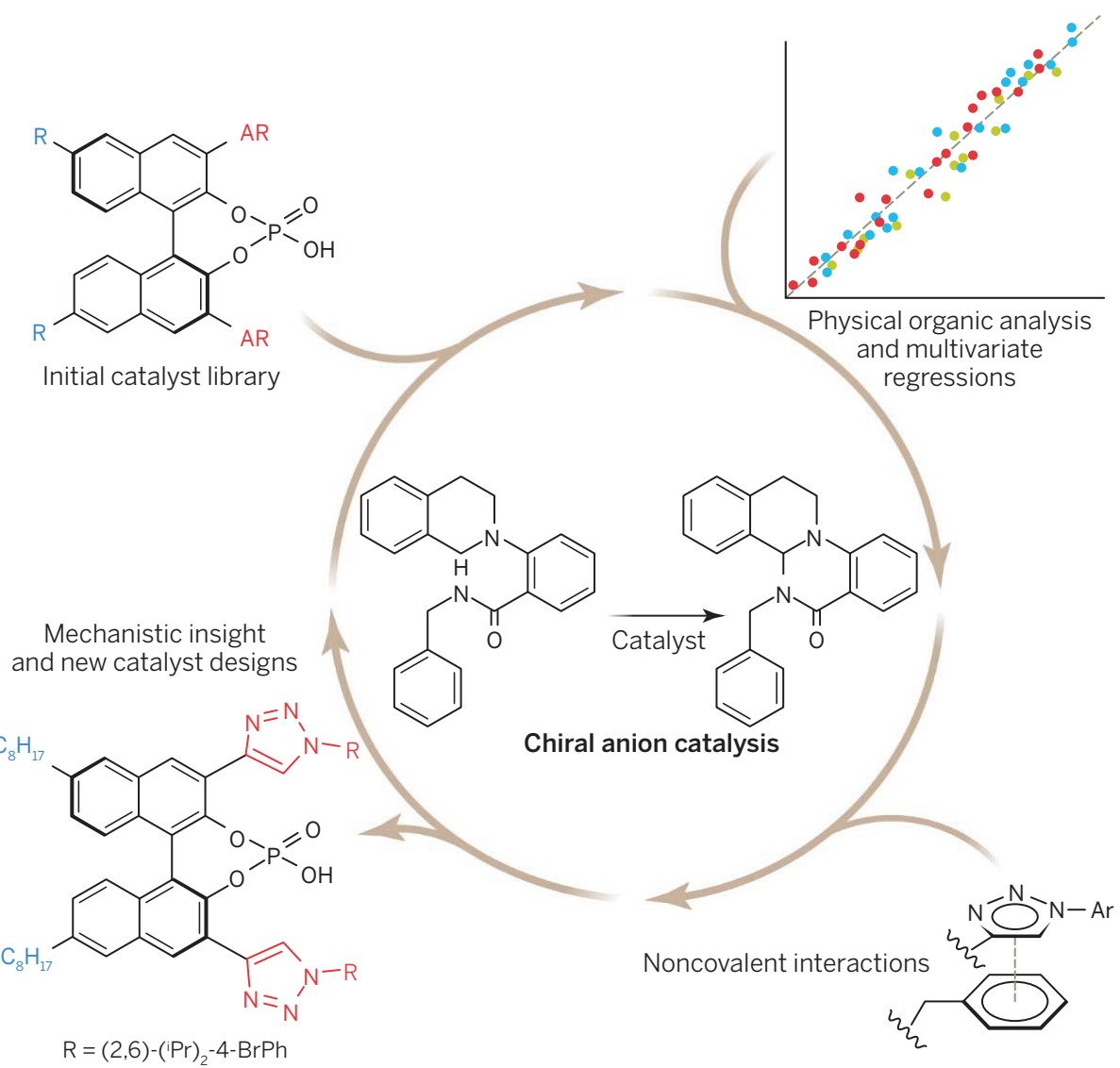

Catalytic design cycle. Screening of an initial catalyst library yields extensive data sets, which are then distilled into predictive mathematical models through multivariate regressions. These models yield new mechanistic insights and lead to improved catalyst designs. Abbreviations: $\mathrm{Pr}$, isopropyl; Ph, phenyl. tion mechanism and the role of nonbonded interactions in these enantioselective reactions, facilitating the rational design of more effective catalysts.

Enantioselectivity typically arises from a difference in free energy between competing transition states that lead to two possible enantiomeric products. Traditionally, such free-energy differences were rationalized by the destabilization of the pathway leading to the undesired product by repulsive steric interactions. However, there has been recent emphasis on the role of favorable noncovalent interactions in enantioselective reactions (2), and more effective catalysts can be designed by preferentially stabilizing one pathway over other, un- 
If you wish to distribute this article to others, you can order high-quality copies for your colleagues, clients, or customers by clicking here.

Permission to republish or repurpose articles or portions of articles can be obtained by following the guidelines here.

The following resources related to this article are available online at www.sciencemag.org (this information is current as of February 17, 2015 ):

Updated information and services, including high-resolution figures, can be found in the online version of this article at:

http://www.sciencemag.org/content/347/6223/718.full.html

A list of selected additional articles on the Science Web sites related to this article can be found at:

http://www.sciencemag.org/content/347/6223/718.full.html\#related

This article cites 15 articles, 1 of which can be accessed free:

http://www.sciencemag.org/content/347/6223/718.full.html\#ref-list-1

This article appears in the following subject collections:

Chemistry

http://www.sciencemag.org/cgi/collection/chemistry 